\title{
Assessing the accuracy of business-level forecasts
}

Pete Brodie, Tullio Buccellato and Eric Scheffel

Office for National Statistics

\section{Summary}

This article presents original work on some aspects of forecasting at the individual business- or firm-level. In particular, two ways are suggested for assessing the accuracy of these forecasts based on the calculation of average percentage errors and the construction of a 95 per cent confidence interval. It is found that the quality of forecasts tend to become increasingly unreliable after two years and that the decay in forecast quality is inversely related to the frequency of the time series - that is the less often a time series is updated/published the faster the deterioration in forecast quality.

\section{Introduction}

Economists routinely generate forecasts of economic variables such as gross domestic product (GDP) and its components. These are important for helping policy-makers and organisations to make decisions about the future. However, forecasts are usually based on published aggregates while, so far, there has been little investigation of the potential for using micro- or business-level data that underlie these aggregates to forecast. This article aims to do just that by using simple time series methods to generate forecasts of business-level data on turnover and capital expenditure as well as suggesting how the accuracy of these forecasts can be assessed.

The main purpose for collecting business-level or micro-data is for the production of economic statistics. However, once these statistics have been published, the remaining value in the microdata is mainly in their use for research. The Virtual Microdata Laboratory (VML) at ONS allows secure access to this data, enabling researchers from government, universities and other organisations to undertake work that focuses on the individual characteristics of businesses and households whilst maintaining data confidentiality.

This work aims to find new value in micro-data. To produce timely aggregated figures for the state of the economy it is usual practise for National Statistics Institutions to impute missing values in business registers. One method for imputing this data to extrapolate past trends using simple one 
variable (or univariate) methods - a process know as nowcasting as the approach is simply attempting to predict the present. Forecasting just takes this one step further, investigating whether past trends in micro-data can be used to predict the future.

Being able to assess the accuracy of these forecasts is important and two ways are proposed. First, estimated points are classed as inaccurate when the margin of error exceeds 20 per cent. A second method implies that the prediction is inaccurate if it falls outside of a 95 per cent confidence interval.

The analysis is conducted on four important ONS business datasets. These are the Annual Business Inquiry ( $A B I)$, the quarterly Capital Expenditure survey (CAPEX), the Monthly Inquiry into Distribution and Services Sector (MIDSS) and the Monthly Production Inquiry (MPI).

The next section of this article outlines the formal framework used to compute forecasts at the individual firm level and how to access their accuracy. The third section presents the results of the empirical analysis and the final section concludes.

\section{Methodological framework}

This section outlines a simple time series procedure, namely the Holt-Winters process that is used to produce forecasts. Then, two methods are proposed for testing the accuracy of out-of-sample forecasts - these are based on mean and median percentage errors and also confidence intervals.

\section{Time series procedures}

A standard multiplicative Holt-Winters process is used to produce forecasts of turnover and capital expenditure for an individual business. This method is already widely-used at the Office for National Statistics (for example in various parts of the Index of Services). It works by using information on the level, trend and seasonality of the time series to form a one period ahead forecast of a variable $\hat{y}_{t+1}$ such that

$$
\hat{y}_{t+1}=\left(m_{t}+b_{t}\right) c_{t-s}
$$

where $m_{t}$ is the level of the series at time $t, b_{t}$ the gradient of the time series and $c_{t-s}$ the multiplicative seasonal coefficient such that $k=1,2, \ldots, s$ for each of the seasons (for example, monthly $s=12$ and quarterly $s=4$ ).

Each of these three coefficients is updated recursively as another observation (or forecast) becomes available, such that: 


\section{Level}

$$
m_{t}=\alpha_{0} \frac{y_{t}}{c_{t-s}}+\left(1-\alpha_{0}\right)\left(m_{t-1}+b_{t-1}\right)
$$

\section{Trend:}

$$
b_{t}=\alpha_{1}\left(m_{t}-m_{t-1}\right)+\left(1-\alpha_{1}\right) b_{t-1}
$$

\section{Seasonal:}

$$
c_{t}=\alpha_{s} \frac{y_{t}}{m_{t}}+\left(1-\alpha_{s}\right) c_{t-s}
$$

The coefficients $\alpha_{0}, \alpha_{1}$ and $\alpha_{s}$ describe how quickly the level, trend and seasonal coefficients are updated in response to new data observations. The higher each of these coefficients, a larger weight is placed on the current observation relative to the past history of the series. These coefficients can be estimated using the past time series. These updating coefficients are likely to be lower the better-behaved/less volatile the past data.

The great advantage of this method lies in its simplicity and its ability to take account of the presence of both a time trend as well as a seasonal component in the data, even when these components are experiencing changes through time. Forecasts are simply generated in a onestep-ahead fashion with the Holt-Winters equations re-estimated each time to take account of a new data observation or forecast. The process also allows a large number of forecasts to be produced fairly quickly, which is important when dealing with a large number of firm-level time series.

The Holt-Winters procedure is used for monthly turnover data from MPI and MIDSS and quarterly investment spending from CAPEX. However, for the annual turnover data collected by the ABI, where seasonality is not an issue and the number of observations is smaller, a basic time trend is used for the basis of extrapolating past data.

Applying these univariate methods requires a complete time series so that there are no gaps or missing observations. Large and small firms are treated differently in ONS's sampling frame, with large businesses typically included in every period while smaller businesses are rotated in and out of the sample, so will typically have periods of non-inclusion. This is usually to lessen the administrative burden on smaller companies. As a result, the forecasting exercise here is limited to a select number (ranging from 38 to 50 ) of the largest businesses in each dataset which have been continuously sampled. Larger firms also tend to be less volatile and therefore easier to forecast using these simple time series methods.

Once the forecasts at the business-level have been produced they are then aggregated. 


\section{Accuracy of forecasts}

A succession of one-step-ahead forecasts $\hat{y}_{i, t+h}$ can be formed for each of the $\mathrm{i}=1, \ldots, \mathrm{N}$ firms, but as the data moves further away from the actual reference time $t$ (that is as $h$ gets larger) the predictive power tends to fall. But how long does it actually take for data to become 'obsolete' in the sense that the quality of the predictive values falls below an acceptable level?

Two approaches are used to assess the quality of forecasts.

\section{Mean and median absolute percentage error}

First, prediction accuracy is summarised with the mean and median absolute percentage errors (MAPE and MedAPE) for the $\mathrm{N}$ firms.

$$
\begin{aligned}
& M A P E_{t+h}=\frac{1}{N} \sum_{i=1}^{N} \frac{\left|y_{i, t+h}-\hat{y}_{i, t+h}\right|}{y_{i, t+h}} * 100 \\
& \operatorname{MedAPE}_{t+h}=\frac{\left|y_{i=N / 2, t+h}-\hat{y}_{i=N / 2, t+h}\right|}{y_{i=N / 2, t+h}} * 100
\end{aligned}
$$

This allows conclusions to be drawn on accuracy in a similar way to what is usually done in National Statistics Institutions with sampling errors.

\section{Confidence intervals}

Secondly, upper and lower bounds around the forecasted values are constructed showing a 95 per cent confidence interval. A forecast is judged unreliable if it falls outside of this range. There are two ways in which the 95 per cent confidence interval can be constructed.

The first is based on fixed upper and lower boundaries calculated with the standard deviation computed at the initial point of the forecasting horizon. Confidence intervals estimated using only the in-sample data can become quite inaccurate as $h$ gets relatively large (that is the forecasts extent into the future). However, results based on this measure are reported because it is based on a real information set. 
The second approach updates these boundaries over the forecasting horizon, computing the standard deviation iteratively as new data points (from forecasts and observed values) emerge so uses both in-sample and out-of-sample information. The variance is expected to grow reflecting a gradual loss in forecasting accuracy over time, so it is anticipated that the confidence bands would widen.

\section{Empirical analysis on ONS business data}

This section presents results from the forecast exercises on the Annual Business Inquiry (ABI), the Capital Expenditure survey (CAPEX), the Monthly Inquiry into Distribution and Services Sector (MIDSS) and the Monthly Production Inquiry (MPI) relating to turnover and capital expenditure. In each case the Holt-Winters or trend extrapolation procedure is fitted but not to the end of the sample. This then allows a number of observations for the accuracy of forecasts to be evaluated. In the case of $A B I, M P I$ and MIDSS turnover five years of observations at the end of the sample are reserved for out-of-sample testing. For investment spending on the quarterly CAPEX survey three years of data are used for testing the accuracy of predictions.

\section{Absolute percentage errors}

Figures 1 to 4 compute the MAPE and MedAPE for the available cross-section of businesses at any given point in time in each survey. This is an equivalent approach to computing of the conditional forecast accuracy of out-of-sample predictions of individual firms. Unsurprisingly, all of the line graphs generally exhibit a positive slope which implies a decreasing forecasting accuracy as a greater out-of-sample prediction horizon is considered. Forecasts of the CAPEX survey (Figure 2) are particularly bad, perhaps due to the inherent volatility in investment data - especially at the firm-level.

Because the MedAPE considers the central rather than the average forecast error it is more robust to outliers. Therefore, significant differences between the MAPE and MedAPE are usually accounted for by a small number of particularly bad forecasts at the individual business level. For example, consider the predictive accuracy measures computed for the last year of the ABI (Figure 1 ), where a large reported turnover value in a single business caused the MAPE to jump from below 50 per cent in 2006 to over 90 per cent in 2007.

Figures 3 and 4 show the conditional forecast accuracy based on the MAPE and MedAPE statistics for the MPI and MIDSS micro-data. These also shows a widening gap between the MedAPE and the MAPE in the last third of the out-of-sample forecast horizon - which is also predominantly due to the influence of one or a few businesses, whose forecasts turn out to be comparatively bad relative to the actual turnover outcome. It is also clear from the monthly forecasts for MPI and MIDSS that not all of the seasonality present in the individual business' turnover series is modelled satisfactorily, as characteristic peaks and troughs are plainly apparent at regular intervals in Figures 3 and 4. 


\section{Figure $1 \quad$ Absolute percentage error - $\mathrm{ABI}$ (turnover)}

Per cent

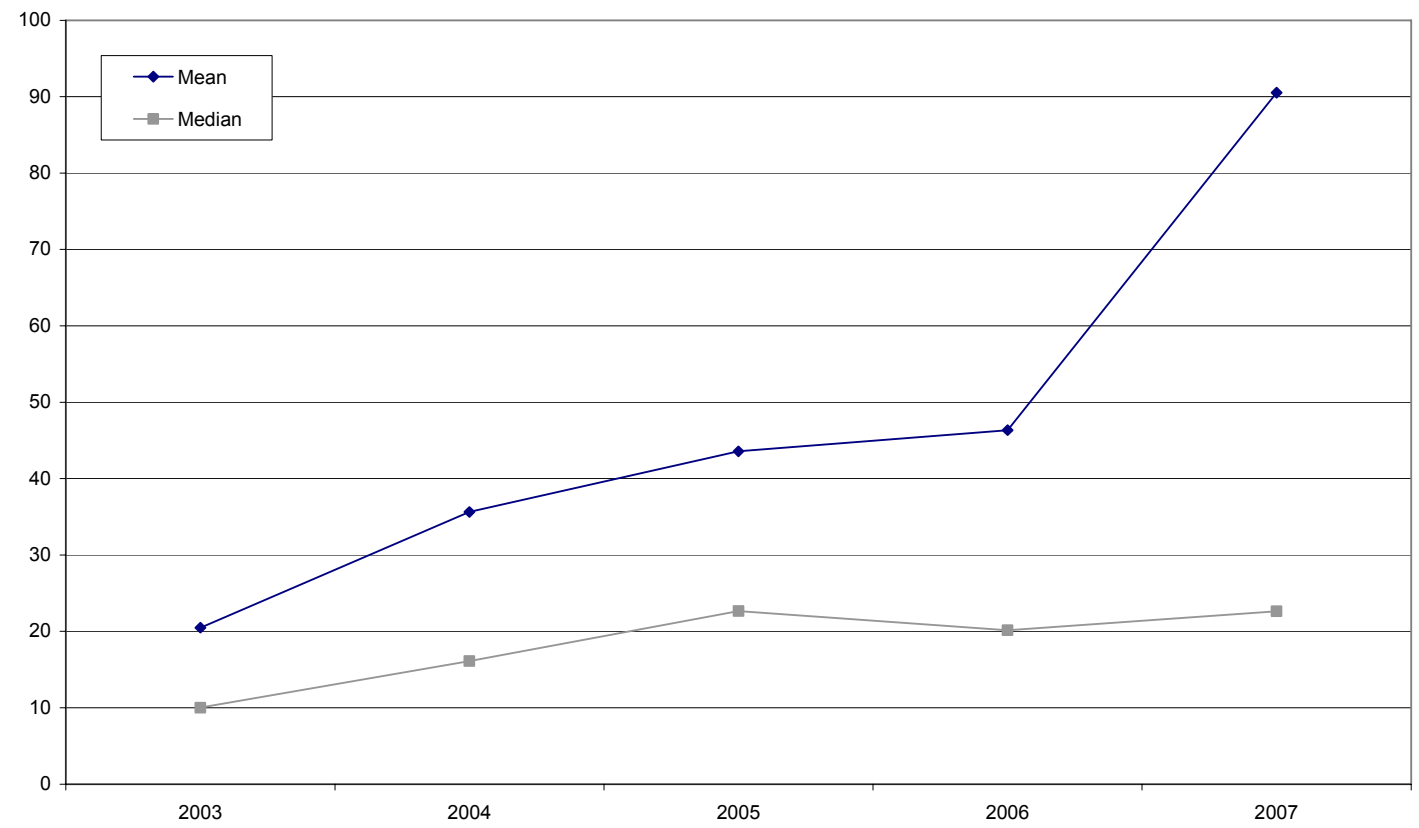

Source: Authors' calculations

Figure 2 Absolute percentage errors - CAPEX (investment)

Per cent

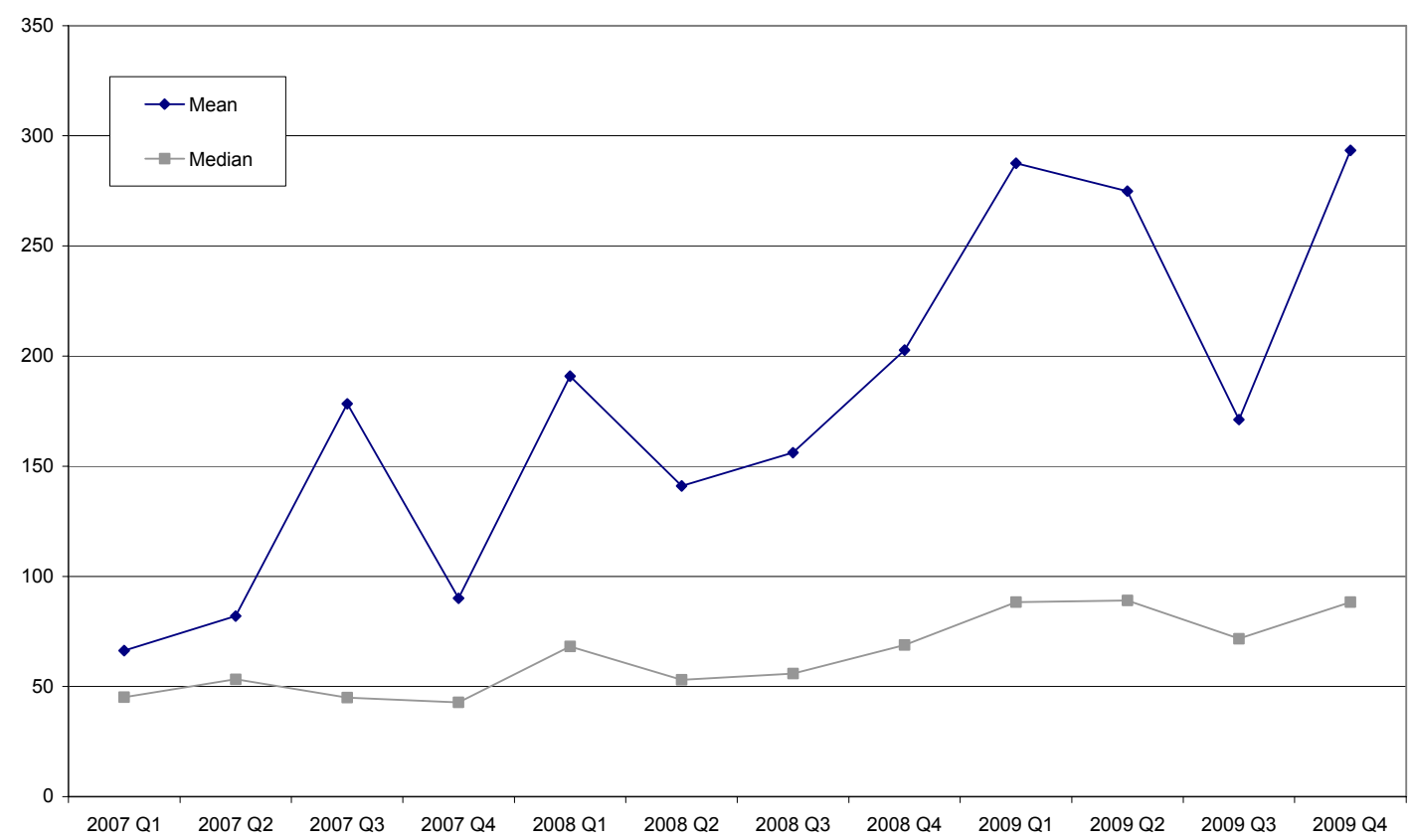

Source: Authors' calculations 
Figure 3 Absolute percentage errors - MIDSS (turnover)

Per cent

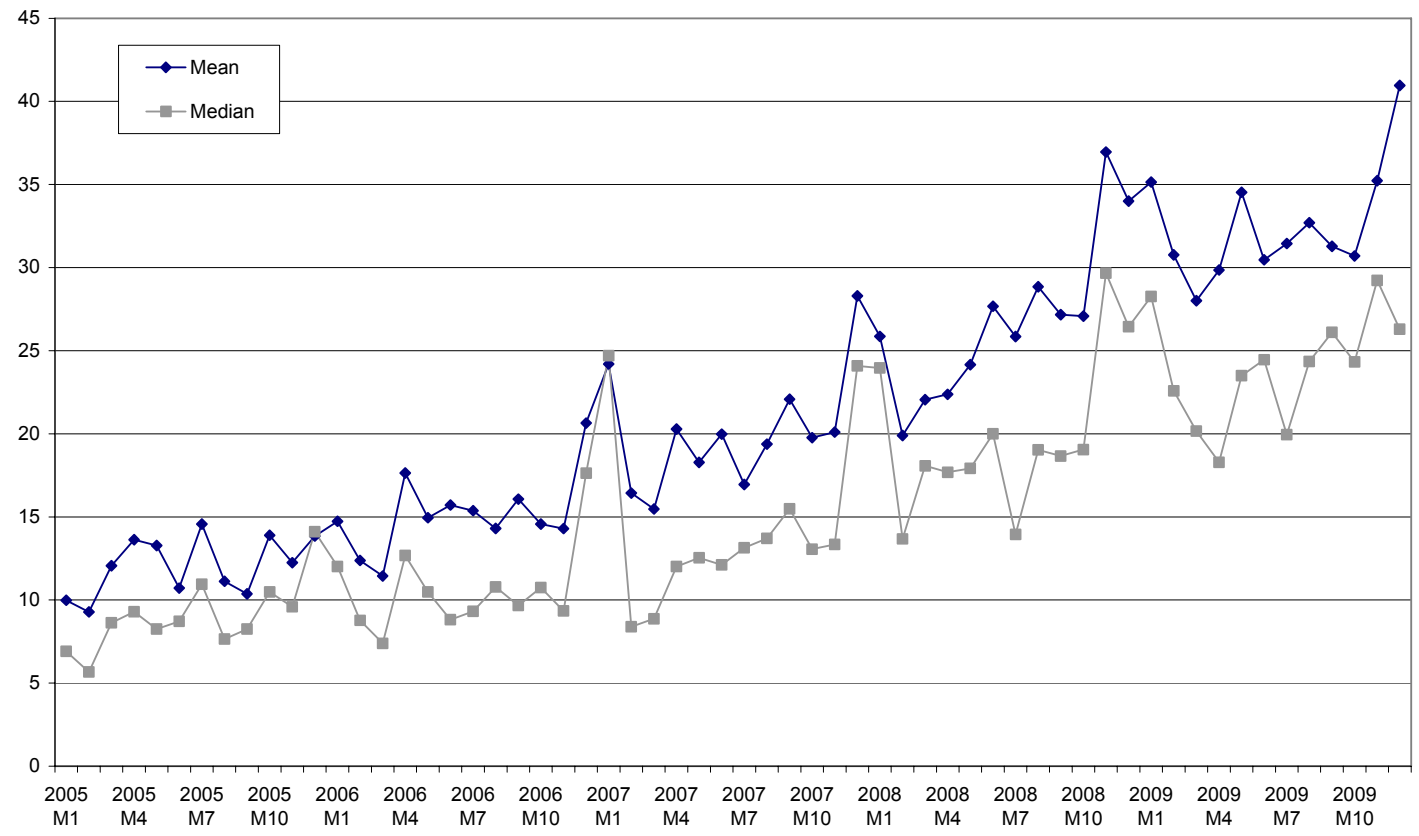

Source: Authors' calculations

\section{Figure $4 \quad$ Absolute percentage errors - MIP (turnover)}

Per cent



Source: Authors' calculations 
In the Annex, Figures A1 to A4 show the unconditional distribution of the MAPE. In each survey, these exhibit very strong right hand side tails which tends to support the argument that divergence between MAPE and MedAPE is driven by a small number of particularly bad forecasts towards the end of the out-of-sample period.

Figure 5 provides a summary of Figures 1 to 4 where a forecast is considered acceptable providing the absolute percentage error is less than 20 per cent. Based on an assessment using MAPE, MIDSS and MPI forecasts are acceptable for around two years, whereas MAPEs for CAPEX and ABI are at no point are below this 20 per cent threshold. MedAPEs, for the argument already given, are generally lower than the respective MAPEs and hence the forecasts are generally below the 20 per cent threshold for longer. The MedAPEs for both MIDSS and MPI are generally below this threshold until nearly the end of the fourth year of forecasts. Out-of-sample $\mathrm{ABI}$ forecasts are now acceptable for two years, however CAPEX forecasts remain unreliable throughout the evaluation period.

\section{Figure $5 \quad$ Summary of MAPE and MedAPE tests}



Source: Authors' calculations

\section{Confidence intervals}

The second approach to assessing forecast accuracy is to calculate at each point in time a 95 per cent prediction interval. The confidence interval can be calculated based on in-sample variances (fixed variance approach) or also to include a rolling variance calculation over the forecast horizon which is updated each time a new forecast is evaluated against the outturn (recursive variance approach).

Figures 6 to 9 display the results. In each case, part (a) of the respective figure shows confidence intervals based on fixed variances and part (b) based on recursive variances. 


\section{Figure 6 Point and density* forecasts - ABI (turnover)}

(a) Fixed variance

Value (aggregate over 50 firms)

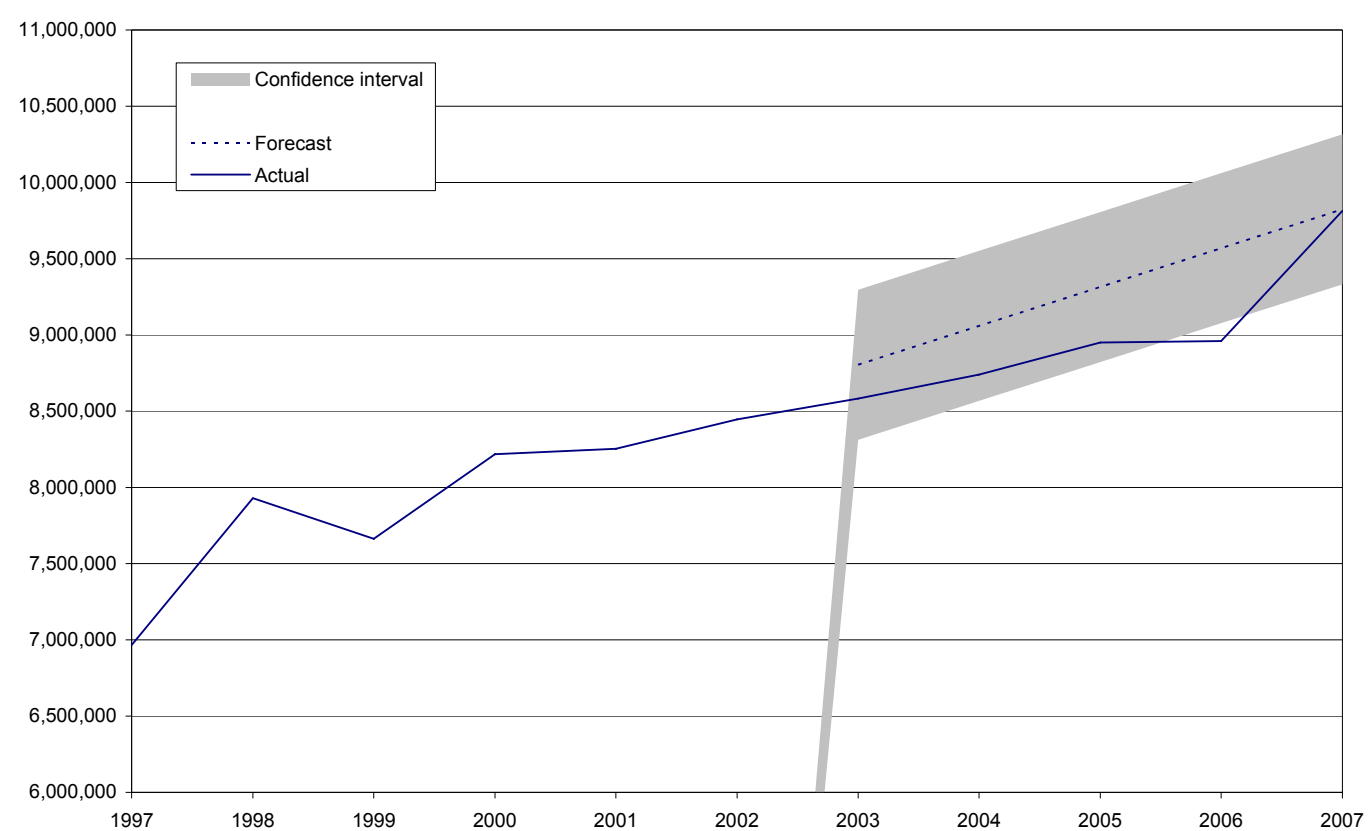

\section{(b) Recursive variance}

Value (aggregate over 50 firms)



* $95 \%$ confidence interval

Source: Authors' calculations 


\section{Figure $7 \quad$ Point and density* forecasts - CAPEX (investment)}

(a) Fixed variance

Value (aggregate over 50 firms)

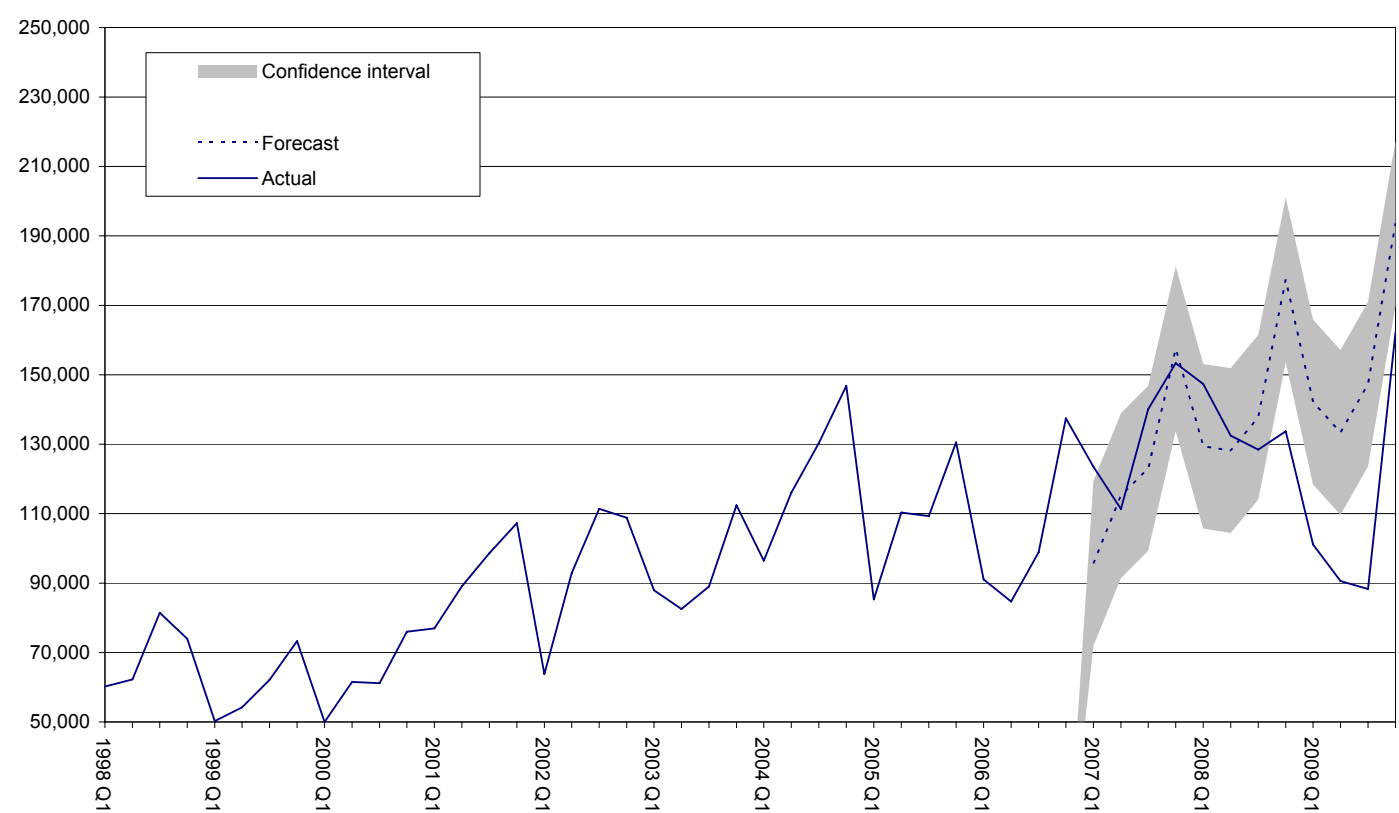

(b) Recursive variance

Value (aggregate over 50 firms)

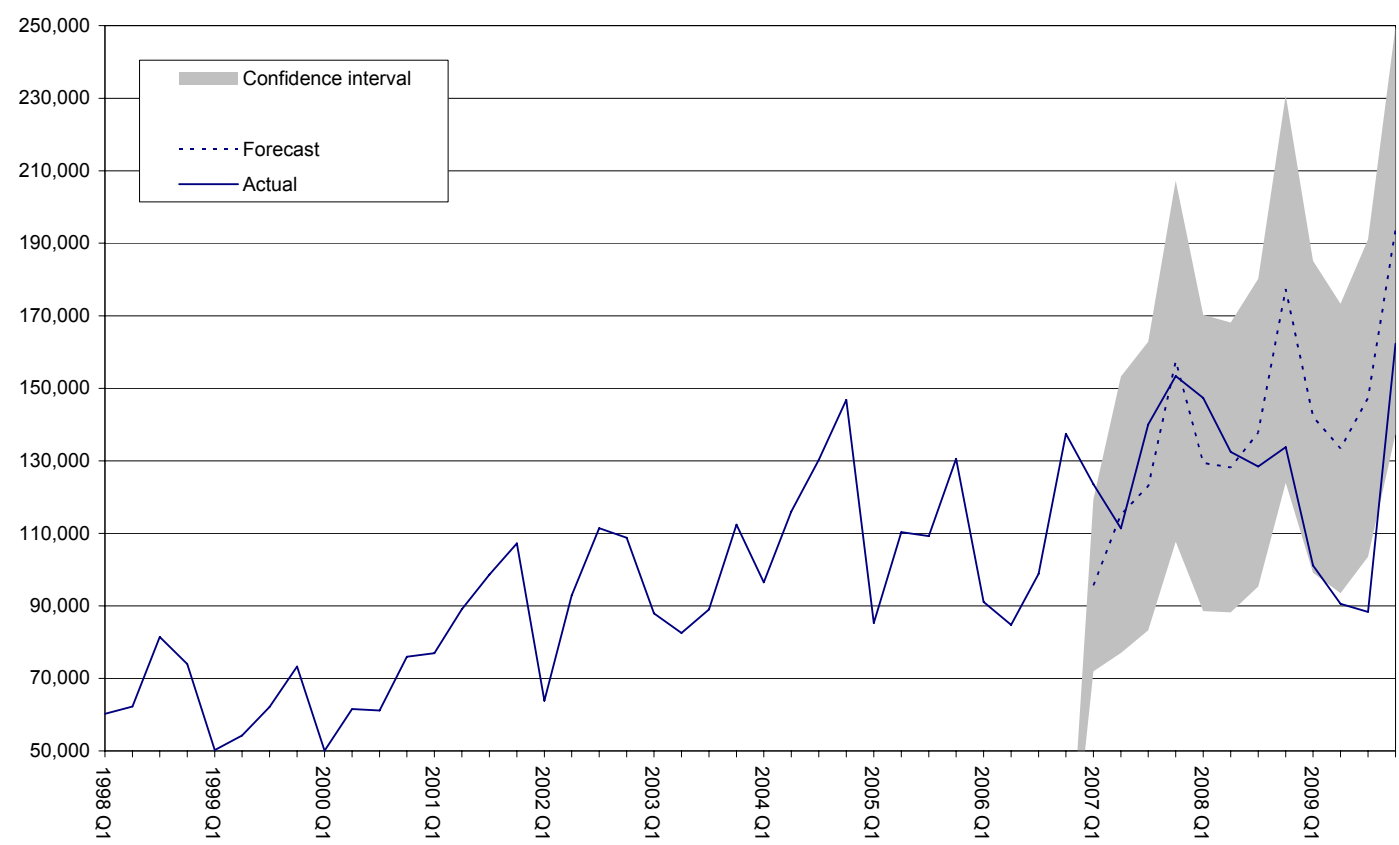

* $95 \%$ confidence interval

Source: Authors' calculations 
Figure $8 \quad$ Point and density* forecasts - MIDSS (turnover)

(a) Fixed variance

Value (aggregate over 50 firms)

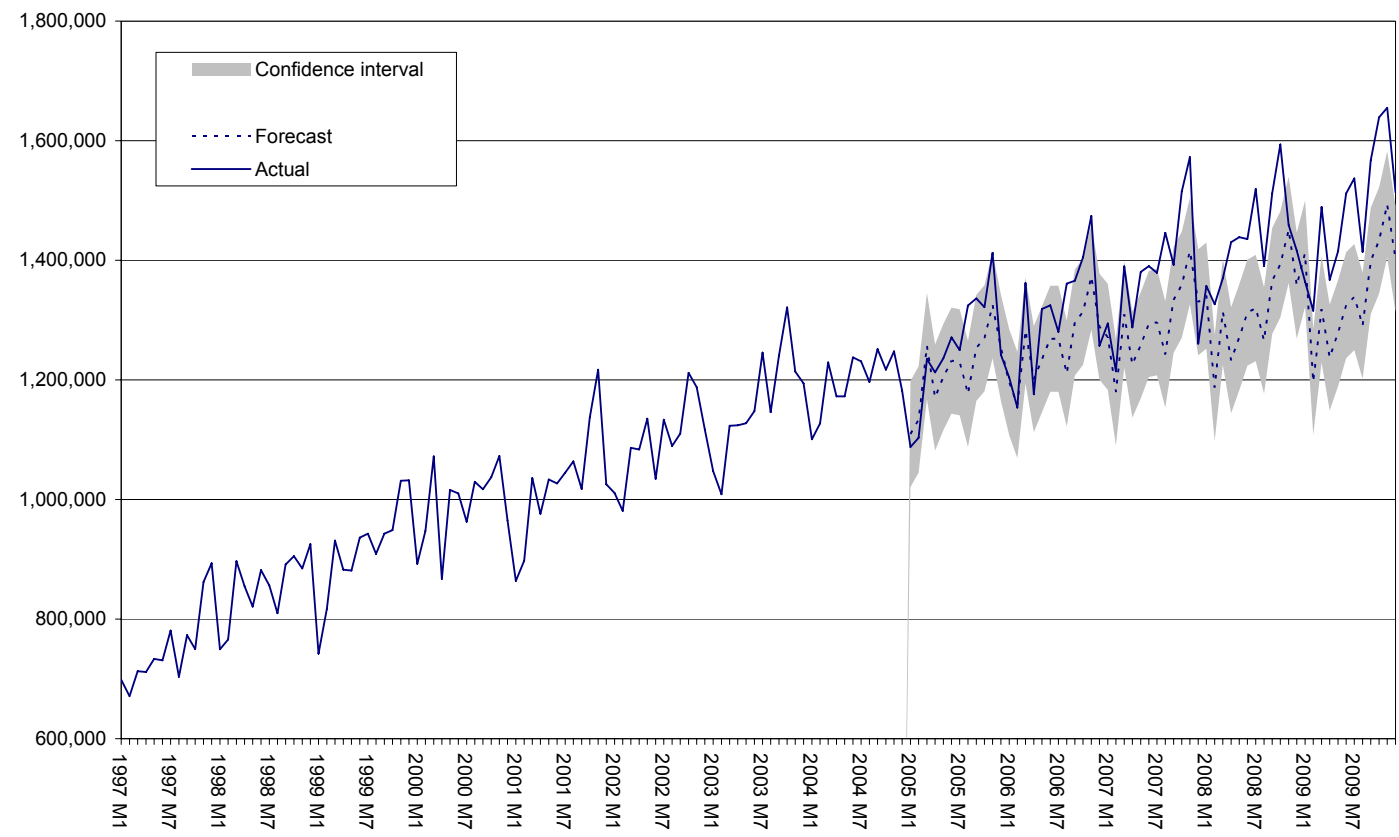

(b) Recursive variance

Value (aggregate over 50 firms)

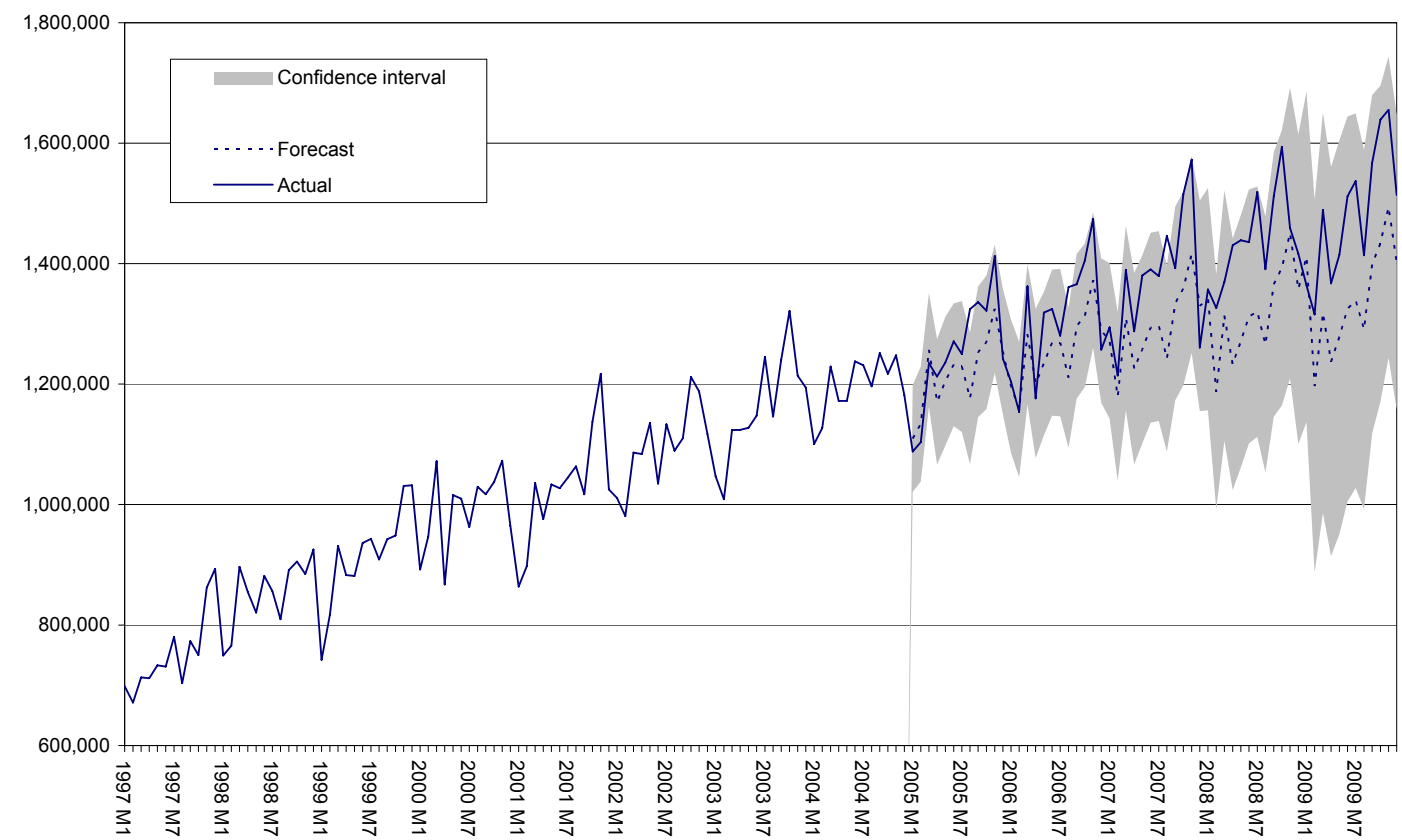

* 95\% confidence interval

Source: Authors' calculations 


\section{Figure 9 Point and density* forecasts - MPI (turnover)}

(a) Fixed variance

Value (aggregate over 50 firms)

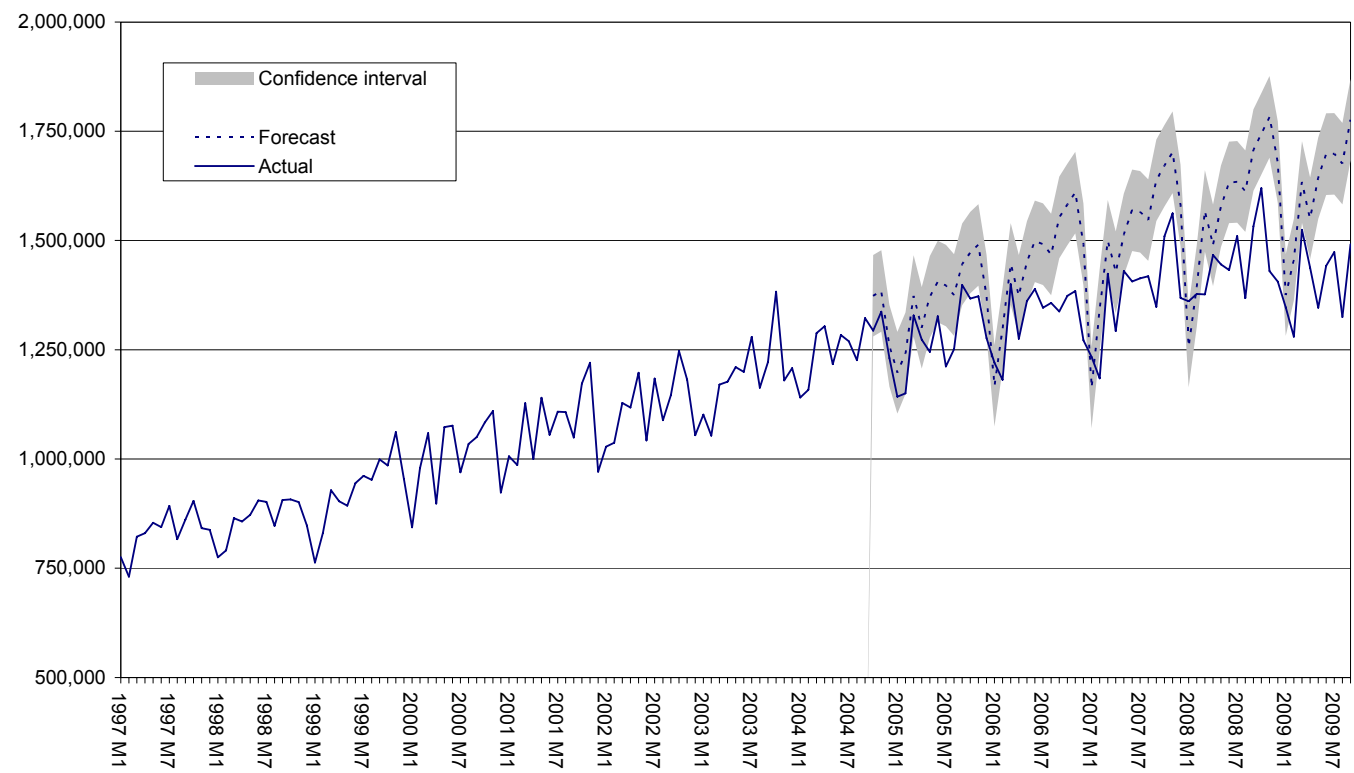

(b) Recursive variance

Value (aggregate over 50 firms)

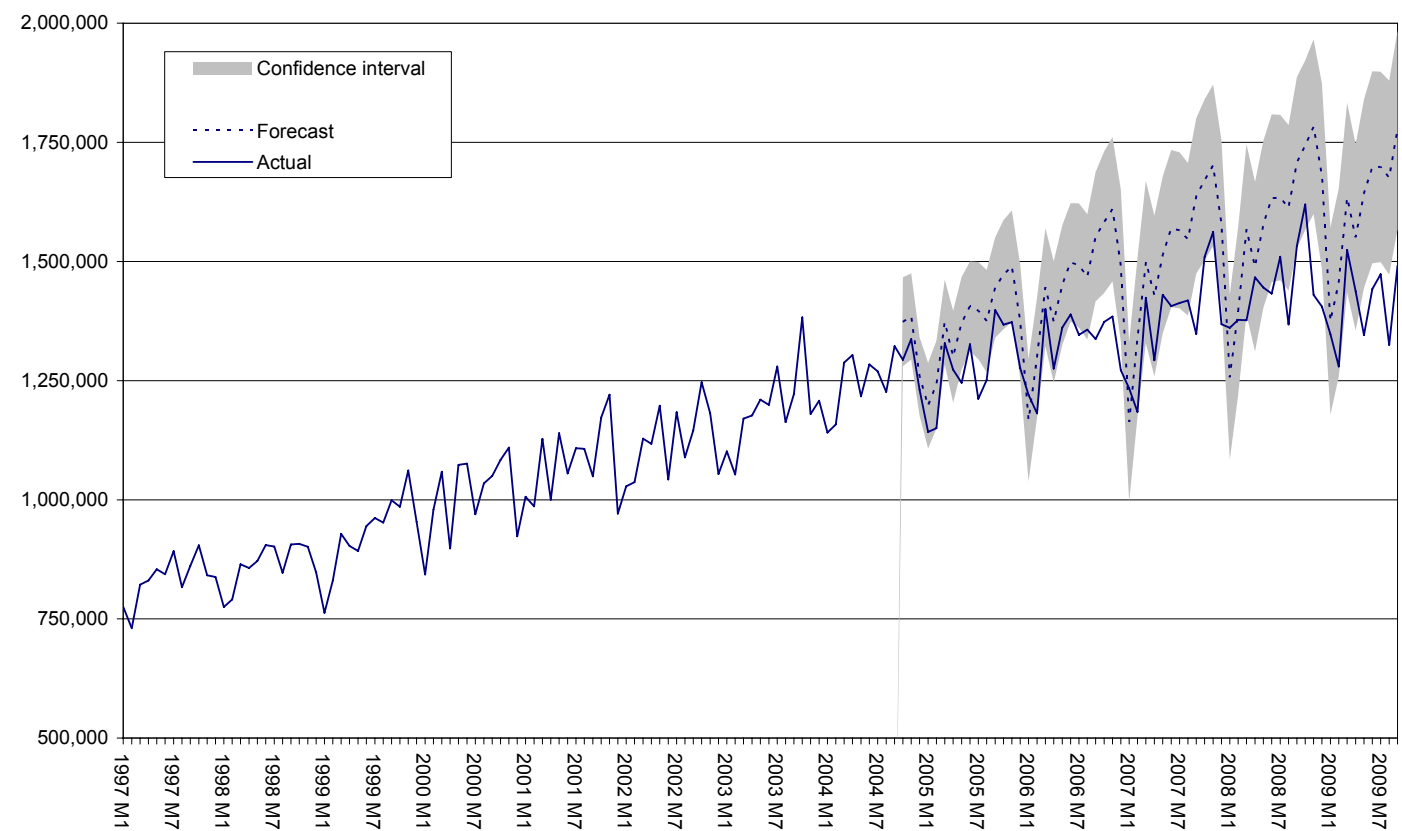

* $95 \%$ confidence interval

Source: Authors' calculations 
Figure 10 provides a summary of the main results shown in Figures 6 to 9 . Different colours have been used to highlight the degree of accuracy of the forecast with respect to the prediction interval - green if completely within the intervals, amber if the difference between the closest interval boundary and the standardized actual value is less than two percent and red when it is outside of the confidence interval.

\section{Figure 10 Summary of forecast findings from Figures 6 to 9}



Source: Authors' calculations

Generally, forecasts perform better on the recursive variance as the 95 confidence interval tends to widen over the forecast horizon, making it easier for the outturn to fall within the prediction interval. On the fixed variance basis, outturns for MPI, MIDSS and CAPEX start to routinely fall outside the 95 per cent confidence interval after two years and three years for $A B I$. The improved performance of CAPEX and ABI forecasts, relative to the absolute percentage error tests, is primarily down to the size of the confidence intervals reflecting the inherent volatility in the underlying data. For instance, quarterly CAPEX data tends to be relatively volatile, so the 95 per cent confidence interval will be calculated to be relatively wider.

\section{Conclusions}

The main objective of this work is to explore the predictive power of individual firms' turnover and capital expenditure series based on four key business surveys administered by the Office for National Statistics. These are the monthly MPI and MIDSS, the quarterly CAPEX and the annual $A B I$. The results suggest that the predictive power of the data starts to drop substantially after the end of the second year and the beginning of the third. However, the rate of decay is not continuous over the forecasting horizon and this seems to be particularly true for annual data (the decay is slower when referring to the rolling forecast). As a result, it is concluded that forecast precision is not independent of the frequency of the data for which such forecasts have been produced. In general, the lower the frequency the slower the deterioration in the forecasts, and vice versa. 
CAPEX forecasts based on quarterly data tend to perform fairly badly and forecast precision declines much faster than the monthly MPI and MIDSS turnover series. This is probably because investment data is much more volatile or lumpy compared to the generally much more stable turnover data taken from the monthly surveys.

\section{Contact}

elmr@ons.gov.uk 
Annex - Unconditional MAPE distributions of MPI, MIDSS, CAPEX and ABI

Figure A1 Distribution of absolute percentage errors - MPI

Relative frequency

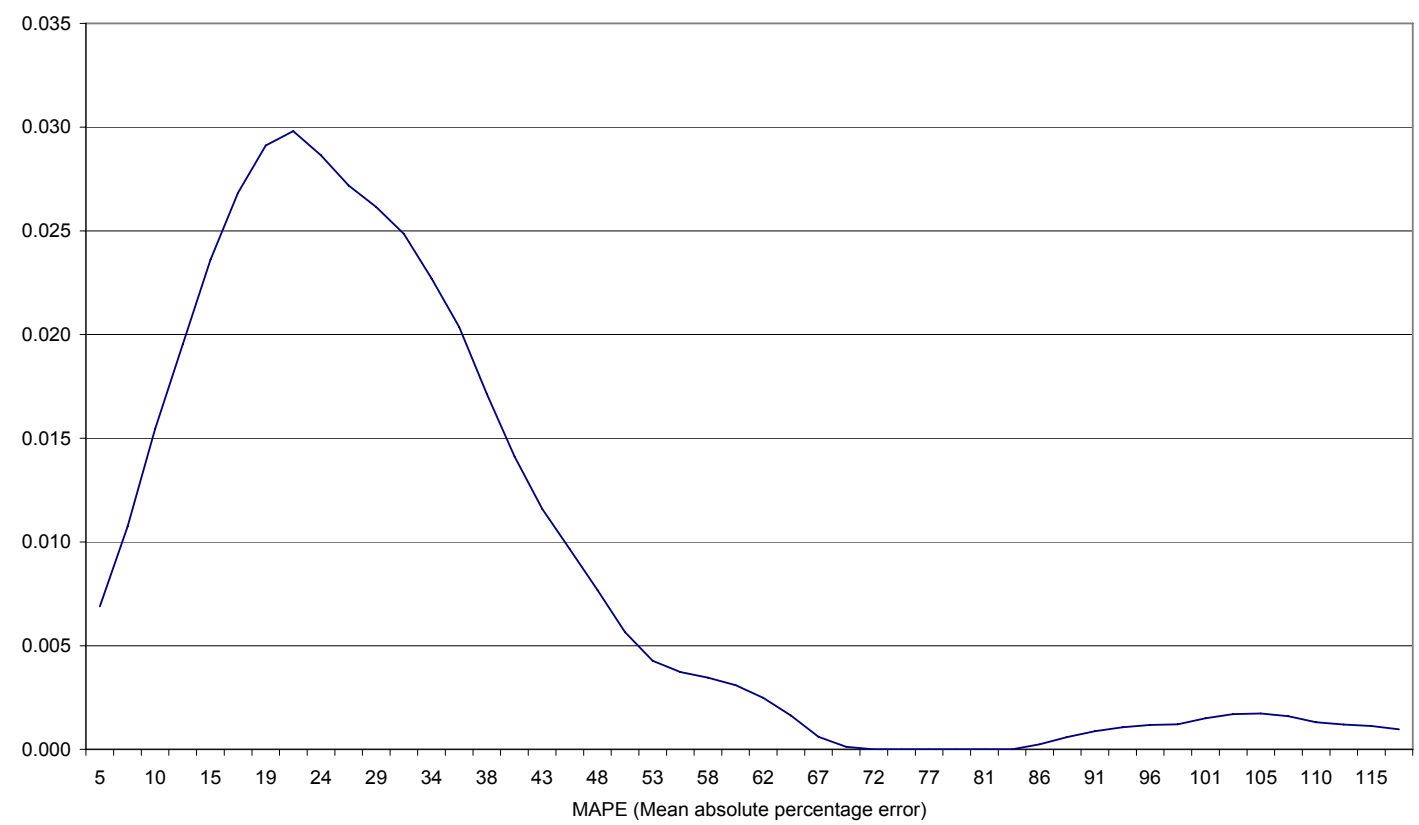

Turnover; Range: 3\% - 120\%; Mean - 30\%; Mode - 22\%

Source: Authors' calculations

Figure A2 Distribution of absolute percentage errors - MIDSS

Relative frequency

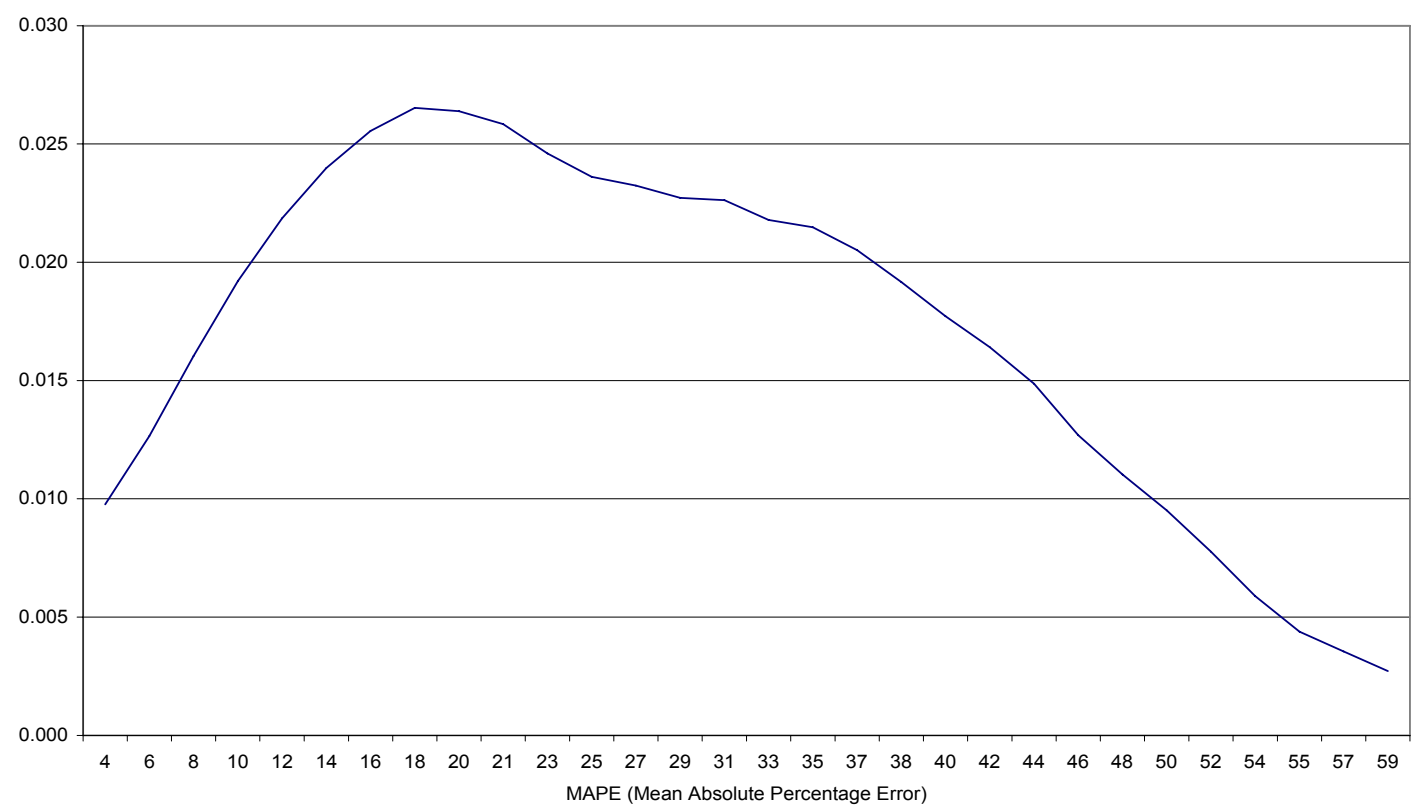

Turnover; Range: $3 \%-60 \%$; Mean - 27\%; Mode - $18 \%$

Source: Authors' calculations 


\section{Figure A3 Distribution of absolute percentage errors - CAPEX}

Relative frequency

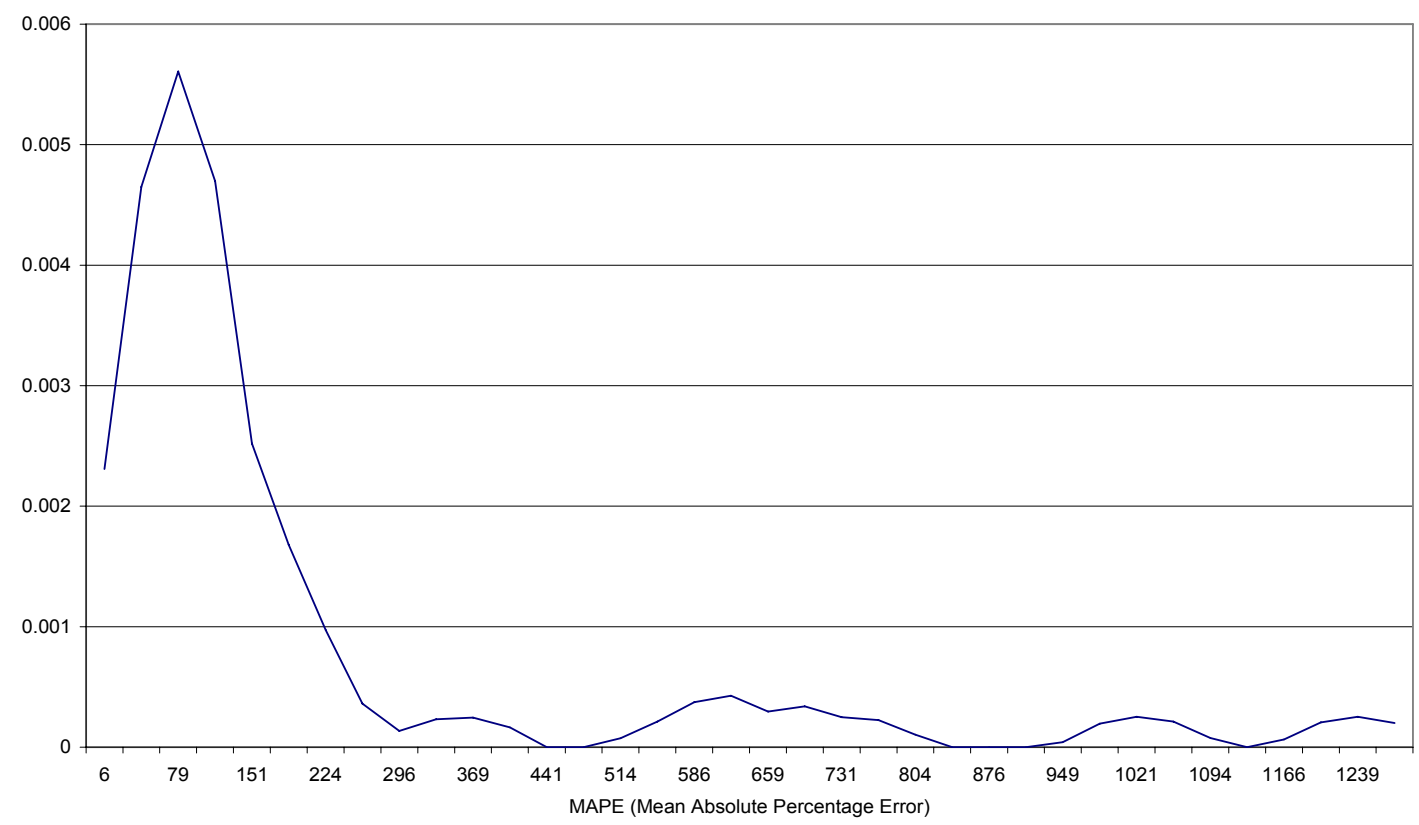

Investment; Range: 40\% - 1200\%; Mean - 207\%; Mode - $80 \%$

Source: Authors' calculations

Figure A4 Distribution of absolute percentage errors - ABI

Relative frequency

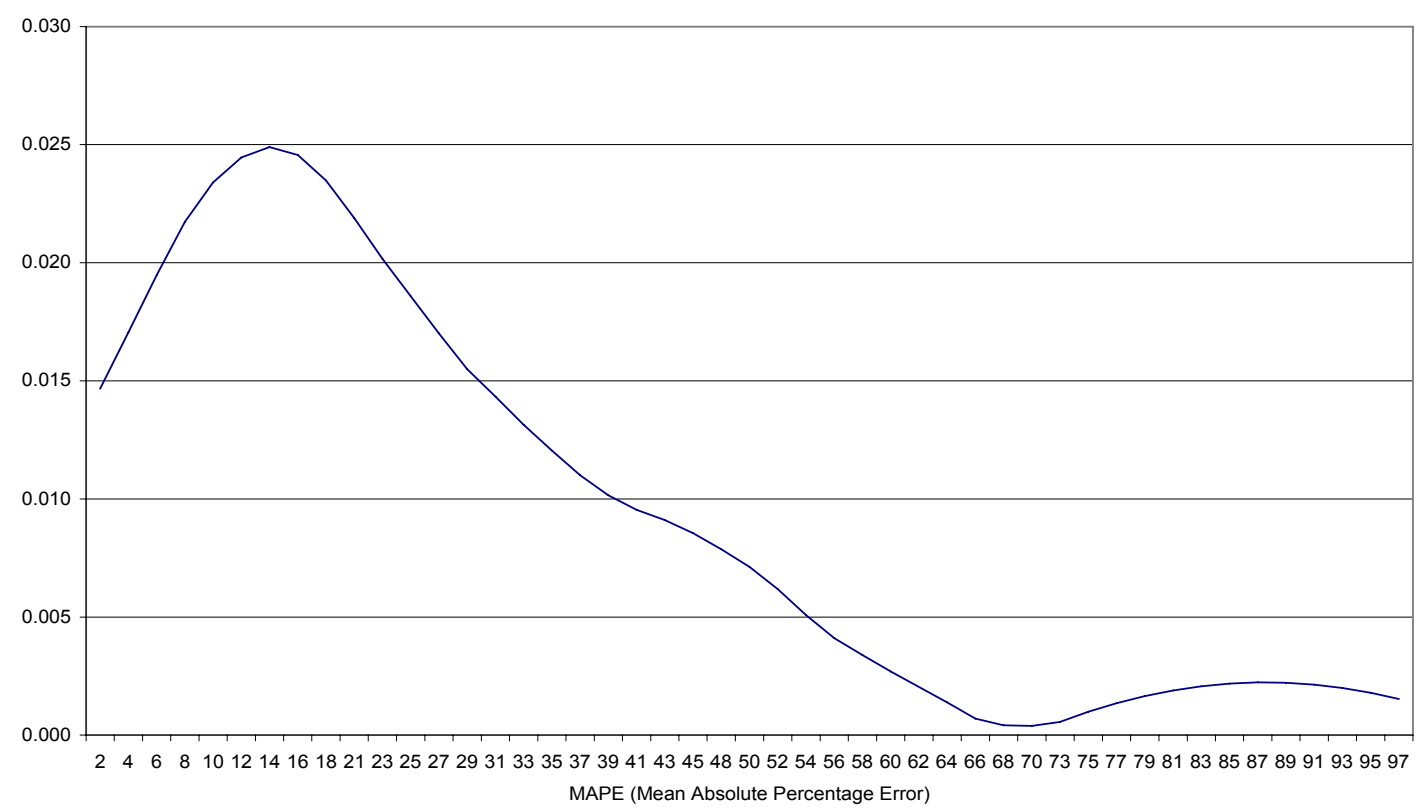

Turnover; Range: $2 \%$ - 100\%; Mean - 20\%; Mode - 14\%

Source: Authors' calculations 